\title{
Accuracy of Fully Automatic vs. Manual Planning of Cardiac MR Acquisitions
}

\author{
M.G. Danilouchkine, J.J.M. Westenberg, H.J. Lamb, J.H.C. Reiber, and \\ B.P.F. Lelieveldt \\ Division of Image Processing, Department of Radiology, C2S-1, \\ Leiden University Medical Center, P.O.Box 9600, 2300RC, Leiden, \\ The Netherlands \\ M.G.Danilouchkine@lumc.nl
}

\begin{abstract}
In this paper, a system for fully automatic planning of shortaxis (SA) cardiac MR acquisition is evaluated. Experimental results from 11 patients and 3 healthy subjects show that the difference between the automatic and manual planning was not statistically significant.
\end{abstract}

\section{Introduction}

Magnetic Resonance (MR) Imaging is becoming a very important tool for the evaluation of the cardiovascular system. To obtain the optimal imaging geometry of the heart, a set of so-called SA slices is acquired. These slices are cross-sections of the heart, which are orthogonal to the principal axis of the left ventricle (LV). Finding the optimal position and orientation of the SA slices requires elaborate planning and is a time consuming and knowledge-specific task. Moreover, manual planning is a subject of substantial inter- and intra-observer variability. Therefore, accurate assessment of the cardiovascular system is quite a complex procedure.

In our laboratory we developed a novel system for fully automatic planning of cardiac MR acquisition [1]. Our approach automatically combines information from the scout acquisitions into a three-dimensional data set, analyzes the volumetric data, determines the position of the heart in the thoracic cavity and devises the oblique planes for the optimal imaging of the cardiac chambers. To achieve this, we formalized knowledge about the thoracic anatomy in terms of a model by identifying a set of anatomical organs with profoundly distinct boundaries (e.g. lungs, heart, spleen, etc.). For each of those organs we derived a 3-D analytical template using a set of the hyperquadric primitives. The latter are combined into a tree structure by means of Constructive Solid Geometry to form an analytical template model of the human thorax. The registration of the model against the segmented scout views of a patient is formulated as a coarse-to-fine sequence of the non-linear minimization problems. This procedure yields the position and orientation of the heart within the thoracic cavity. Once the correspondence is established, the spatial position and orientation of the SA imaging planes can be obtained. 


\section{Results}

Cardiac MR data sets were acquired on a 1.5-T MR scanner (Gyroscan Intera, Philips Medical Systems, Best, the Netherlands). The subject population included 11 patients and 3 healthy volunteers. For each subject we acquired the multislice scout views in 3 orthogonal directions (20 transversal, 20 coronal, 20 sagittal) without respiratory and cardiac triggering using the whole body coil. Multislice, multi-phase SA views were acquired with cardiac and respiratory triggering using the dedicated cardiac coil. The SA views were planned by the experienced operator who weekly conducts $3-4$ cardiac examinations.

For quantitative comparison of the accuracy of the automatic vs manual planning we derive the criterion based on the angular deviation of the geometric LV axis. To determine the orientation of the geometric LV axis, the endocardial borders were manually outlined in the SA slices at the end-diastolic phase. A straight line was fitted through the centers of gravity of endocardial contours using the least-square approach. This line defines the spatial orientation of the true LV axis. The accuracy of the manual planning was specified as an angle between the LV axis and the spatial orientation of the imaging plane in the SA acquisition. The deviation of the computed and true LV axes was considered as the error of the automatic planning. The degrees of the LV axis deviation from the manually planned (M) and automatically computed (A) LV axes for the patients (P1-P11) and the healthy volunteers (H1-H3) are summarized below.

\begin{tabular}{c||c|c|c|c|c|c|c|c|c|c|c|c|c|c||c|c}
\hline & H1 & H2 & H3 & P1 & P2 & P3 & P4 & P5 & P6 & P7 & P8 & P9 & P10 & P11 & Mean & Std.Dev \\
\hline \hline A & 7.3 & 9.2 & 7.8 & 2.1 & 12.4 & 6.8 & 3.6 & 5.0 & 12.5 & 4.5 & 10.3 & 15.7 & 4.0 & 14.8 & 8.3 & 4.3 \\
\hline $\mathrm{M}$ & 7.4 & 0.9 & 10.1 & 8.8 & 9.4 & 7.5 & 6.4 & 4.1 & 4.7 & 5.7 & 6.6 & 3.7 & 6.7 & 13.7 & 6.8 & 3.1 \\
\hline
\end{tabular}

\section{Discussion}

The accuracy of the automatic vs manual planning of cardiac MR acquisitions was evaluated. The average LV axis deviation amounts to 6.8 for the manual and 8.3 for the automatic procedure. Although the automatic planning tends to be slightly less accurate, this method does not suffer from inter- and intraobserver variability. Moreover the paired t-test revealed that the average LV axis deviations are not significantly different. Additionally, the automatic planning is more time efficient (30 seconds for automatic vs 5-10 minutes for experienced operator) and less knowledge-specific.

Acknowledgement. This research is supported by LUMC revolving fund and STW grant LPG5651.

\section{References}

1. Lelieveldt, B. P. F., Sonka, M., Bolinger, L., et.al: Anatomical Modeling with Fuzzy Implicit Surface Templates: Application to Automated Localization of the Heart and Lungs in Thoracic MR Volume. CVIU 80 (2000) 1-20 